\title{
Ethnologies
}

Collections - Collectionneurs. Textiles d'Amérique et de France. Par Jocelyne Mathieu et Christine Turgeon, dirs. (Québec : Célat et Presses de l’Université Laval, 2002. Pp. 240, ISBN

2-7637-7856-9)

Le fil de l'art. Les broderies des Ursulines de Québec. Par Christine Turgeon (Québec : Musée du Québec et Musée des Ursulines, 2002. Pp. 155, ISBN 2-551-21530-7)

\section{Anne-Hélène Kerbiriou}

Volume 26, numéro 1, 2004

Littératie

Literacy

URI : https://id.erudit.org/iderudit/013356ar

DOI : https://doi.org/10.7202/013356ar

Aller au sommaire du numéro

Éditeur(s)

Association Canadienne d'Ethnologie et de Folklore

ISSN

1481-5974 (imprimé)

1708-0401 (numérique)

Découvrir la revue

Citer ce compte rendu

Kerbiriou, A.-H. (2004). Compte rendu de [Collections - Collectionneurs. Textiles d'Amérique et de France. Par Jocelyne Mathieu et Christine Turgeon, dirs.

(Québec : Célat et Presses de l'Université Laval, 2002. Pp. 240, ISBN

2-7637-7856-9) / Le fil de l'art. Les broderies des Ursulines de Québec. Par

Christine Turgeon (Québec : Musée du Québec et Musée des Ursulines, 2002.

Pp. 155, ISBN 2-551-21530-7)]. Ethnologies, 26(1), 260-267.

https://doi.org/10.7202/013356ar d'utilisation que vous pouvez consulter en ligne. 
Cette approche mériterait d'être appliquée à d'autres textes de la littérature de contact, ce qui permettrait d'éclairer le rapport très intéressant entre le concept de l'altérité et la politique coloniale.

Stéphanie Chaffray CÉLAT et Université Paris IV Sorbonne

Collections - Collectionneurs. Textiles d'Amérique et de France. Par Jocelyne Mathieu et Christine Turgeon, dirs. (Québec : Célat et Presses de I'Université Laval, 2002. Pp. 240, ISBN 2-7637-7856-9)

Le fil de l'art. Les broderies des Ursulines de Québec. Par Christine Turgeon (Québec : Musée du Québec et Musée des Ursulines, 2002. Pp. 155, ISBN 2-551-21530-7)

« ... filé plein, lame, filé riant, point lancé empiétant polychrome »; " lampas fond satin blanc, broché soie, chenille, filé et frisé or »; ou encore «velours ciselé deux-corps », «broderies au passé plat, point de tige et point de sable polychrome ${ } . .$.

Si ce n'était que pour le vocabulaire délicat des « artistes à l'aiguille », la finesse d'exécution, voire les prouesses techniques qui se découvrent au fil des broderies anciennes, l'ouvrage CollectionsCollectionneurs vaudrait déjà bien le détour. Mais les désignations si fines de cet ouvrage spécifiquement voué aux textiles, des parements d'autels aux teintures, des courtepointes aux dentelles, nous font aussi entrer de plain-pied dans une petite partie du vaste monde que l'on appelle familièrement les «arts déco ».

Dans les musées, les collections de textiles, volumes gigantesques de pièces fragiles, entre le beau et l'utile, au carrefour des objets d'art et des objets ethnographiques (si tant est qu'il faille distinguer entre les deux, ce que cet ouvrage se garde de faire), soulèvent des questions particulières, en Amérique du Nord et en France. Le problème le plus crucial en ce domaine est que les collections, particulièrement volumineuses, se trouvent être très insuffisamment inventoriées et documentées.

Cet ouvrage, dirigé par Jocelyne Mathieu et Christine Turgeon, confronte les questionnements qui découlent de ce problème essentiel sur une grande échelle. Ce sont les actes du colloque éponyme, 
exclusivement voué aux textiles, qui se trouvent rassemblés ici; fait assez rare, car la plupart du temps les spécialistes de ce domaine interviennent ponctuellement dans le cadre de colloques disciplinaires différents. Les rassembler permettait de rapprocher des traditions d'approche complémentaires : "les conférenciers français avec leur expérience dans l'analyse des pièces, la publication de catalogues, la réalisation de thesaurus et d'inventaires très documentés [et] les participants québécois et canadiens avec la diversité des corpus, l'approche pluridisciplinaire, l'expertise en muséologie et en mise en espace, en restauration et en conservation » (5).

Les intentions de l'ouvrage paraissaient de prime abord suffisamment techniques pour laisser présager pour le moins d'une certaine austérité, d'une orientation réservée aux muséologues spécialistes. Mais Jocelyne Mathieu et Christine Turgeon, après avoir élaboré en introduction un tour d'horizon synthétique et efficace de l'état de la question - et signalé notamment que là, comme ailleurs, s'est effectué pour les chercheurs le passage de l'ethnographie de terroir à l'ethnologie des influences et des métissages (2) - , surprennent très heureusement le lecteur par le choix des articles de ce collectif, dont l'ordonnancement permet une vue d'ensemble de l'extrême variété d'approches de ce monde des textiles et de l'immense variété de ces textiles eux-mêmes.

La première surprise vient de l'article de Nicole Pellegrin, «Les charmes du périssable. Pour une approche anthropologique des collections de textiles européens ». À la recherche des motivations des collectionneurs de textiles, pour comprendre les ressorts profonds de la constitution des collections muséales - chaque collection étant unique et réfractaire à toute " généralisation historicisante » (30) —, elle plonge, littéralement, dans la préconscience et les secrets des collectionneurs privés, littérairement aussi, ce qu'on ne saurait reprocher, dans leurs sensualités et psychés : "[L]es jeux avec les morts, leurs fantômes et leurs anciennes (secondes) peaux — déjà usées mais convoitées, achetées, nettoyées, rassemblées, exposées — sont à la fois des affaires de possession et des rêves de résurrection ». Là où le fétichisme est métaphysique (13), la collection frôle une dimension du sacré. Pour Nicole Pellegrin, chaque collection est un système en soi, "inscrite qu'elle est... dans un temps, dans une morale, dans un ensemble de valeurs esthétiques et dans le psychisme propre à un(e) maître(sse) d'œuvre»(30). 
L'article de Nicole Pellegrin introduit l'idée de la «collection qui est un système en soi », idée qui constitue la ligne de fond de l'ensemble de l'ouvrage, dont le découpage en quatre parties thématiques très différentes permet par ailleurs l'angle de vue global sur la diversité des sujets et des approches.

La première partie, "Amérindiens et premiers contacts », juxtapose deux articles de nature très différente, que l'optique archéologique réunit. «Mi'kmaq textiles », de Joleen Gordon, présente un panorama de la broderie chez les Mi'kmaq, depuis les premiers exemples archéologiques, remontant à 2500 ans, jusqu'à nos jours. En plus de mettre l'accent sur certaines spécificités de techniques ou de matériaux anciens, elle présente des exemples de prolongation de ces spécificités dans des broderies d'inspiration européenne. Si, à la fin du XVIII siècle, les motifs décoratifs du costume mi'kmaq présentaient des influences européennes très nettes, ces influences n'ont pu prendre corps qu'en raison d'une très ancienne et toujours vive tradition de broderie. Les femmes mi'kmaq, dont le savoir-faire s'est transmis à très long terme, par successions et incorporations, ont fini au XIX ${ }^{\mathrm{e}}$ siècle par créer des objets brodés exclusivement pour les Européens, des objets qui n'avaient pas d'usage dans leur propre culture (48). Ce simple panorama de la broderie dans un groupe amérindien se révèle donc finalement un exposé concis de certaines dimensions de la relation à l'autre, échange ou sujétion.

Élise Dubuc, quant à elle, ne questionne pas le cheminement de l'objet dans sa culture, mais bien plutôt l'objet en lui-même dans "Archéologie d'un vêtement historique. Les vêtements basques du XVI ${ }^{\mathrm{e}}$ siècle trouvés au Labrador et leur carrière muséographique ». Son article détaille les multiples questionnements (ainsi que les problèmes techniques) du muséologue se consacrant à reproduire les ultimes vêtements, portant encore l'empreinte des corps, de marins anonymes. Les techniques de "reproduction des vestiges " sont ici un moyen d'analyse (58), cette étape se prolongeant ensuite sous forme d'exposition des vêtements ainsi reconstitués, "du spectral au spectaculaire » (60), c'est-à-dire de ce que la recherche scientifique finit par donner à voir au public. Implicitement, derrière l'aspect scientifique et l'entrée de ces secondes peaux mortuaires dans la "sphère des représentations » (62) se profilent ces « rêves de résurrection» évoqués par Nicole Pellegrin. Cette idée court en sourdine dans l'article de Dubuc; la représentation de ces vêtements passe "par une réelle transsubstantiation $»(60)$. 
Les deux articles évoqués ci-dessus donnent la couleur de l'ouvrage : c'est un jeu d'alternance entre l'ethnographie, l'histoire des influences, des échanges, du jeu des motifs, et les interrogations muséologiques sur des collections dans leur ensemble ou sur des points techniques précis. La deuxième partie, " De la manufacture à l'atelier de broderie ", relève davantage du premier caractère. Elle détaille en cinq articles cinq collections : deux manufacturières — «La manufacture de Georges Le Manach. Une collection utile, une collection en évolution " de Sandrine Bachelier et «De la production à la collection. Les fonds d'archives des fabricants de soieries lyonnais " de Florence Valantin ; deux collections de religieuses — « Collection d'objets ou objets de collection? Les textiles du couvent des ursulines d'Auch (France, 18601897) » de Christine Aribaud et «La collection de la Fondation Abbaye de la Lucerne » de Josiane Pagnon; ainsi qu'une collection relevant de la production d'une personne unique - «Une collection-relique, les ornements brodés de Jeanne LeBer (1662-1714)» de Christine Turgeon.

Cette partie de l'ouvrage est la plus développée, certainement en raison de l'ampleur de ces collections qui permettent pour chacune des études de fond très documentées, autant qu'en raison de la nature même des textiles sur lesquels elles portent (majoritairement des broderies religieuses d'une qualité exceptionnelle). Ici, contrairement à la plupart des collections muséales prises dans leur ensemble, les chercheurs peuvent mettre des noms (ou au minimum discerner des groupes précis) sur de nombreux ouvrages. Chacun de ces groupes a élaboré un style particulier, mais dans chaque cas les chercheurs ont décelé des influences du temps (reçues ou données) pour tous ces textiles, même dans le cas des religieuses les plus retirées du monde, comme Jeanne LeBer. S'il fallait définir une simple impression générale à la lecture de ces articles si documentés, il semble que ce monde des motifs textiles - et plus particulièrement la broderie - soit le plus malléable, le plus perméable aux influences extérieures; on finirait presque aussi par percevoir, dans ces déploiements symétriques de motifs toujours similaires et sans cesse remodelés les mains de celles qui ont réalisé ces peintures à l'aiguille. Monde sensible s'il en est, où les fragiles fils de soie se font fleurs impérissables, non plus « rêves de résurrection » mais fluctuant désir d'éternité.

Moins spectaculaires de par leur nature même, les «Collections textiles et (la) culture populaire » qui font l'objet de la troisième partie ne comptent que trois articles. Ceci est certainement symptomatique 
du nombre de chercheurs qui s'attachent à ce domaine. Assez significativement aussi, tandis que la seconde partie mettait en jeu des collections majoritairement françaises, celle-ci porte exclusivement sur les arts textiles traditionnels québécois. L'intérêt spécifique de ces trois articles est de questionner précisément les connaissances considérées comme acquises sur des ensembles de techniques ou d'objets traditionnels.

Dans «L'analyse de colorants sur des textiles domestiques du XIX siècle ", Louise Lalonger explore le passage des colorants naturels aux colorants synthétiques dans les textiles traditionnels à la fin du XIX siècle. Ces analyses chimiques des produits tinctoriaux sont placées en regard des enquêtes orales de Robert-Lionel Séguin et de Marius Barbeau sur les pratiques régionales (la connaissance des plantes tinctoriales) en ce domaine. La valeur des enquêtes orales est moins remise en question ici que le désir de découvrir des régionalismes : « les textiles traditionnels n'offraient pas de particularités régionales comme on l'a toujours cru... ils reprenaient plutôt une gamme chromatique à partir de produits présents un peu partout dans le monde " (182).

Le « rêve d'authenticité » de l'ethnologie traditionnelle s'en trouve quelque peu mis à mal mais se trouve en quelque sorte prolongé par un « rêve d'intégration » implicitement présent dans l'article suivant : «La floraison de la courtepointe québécoise» de Marie Durand. L'authenticité «purement » québécoise de cet objet traditionnel avait souvent été contestée, mais Durand aborde le problème sous l'angle inverse. La courtepointe n'est effectivement pas une création québécoise ex nihilo, mais a trouvé son point d'aboutissement au bout de deux siècles et demi d'adaptations et d'emprunts. L'auteure conclut cependant sur les traits qui confèrent à cet objet sa spécificité québécoise acquise au fil du temps dans l'ensemble nord-américain.

Cette spécificité québécoise est questionnée sous un autre angle par Nathalie Hamel dans « La collection du ministère de l'Agriculture. Un témoignage du renouveau artisanal au Québec ». Dans une entreprise volontariste, le ministère de l'Agriculture avait incité au début du $\mathrm{XX}^{\mathrm{e}}$ siècle les Cercles de Fermières à (re)créer intensivement le tissage et les travaux textiles traditionnels dans un esprit «canadienfrançais » : des productions " authentiques » massivement orchestrées. Mais les choix qui ont présidé à l'élaboration de la collection du ministère laissent l'auteur perplexe, cette volonté de revalorisation se jouant sur 
une grande ambivalence : valorisation du «typique » au moyen de modèles étrangers choisis pour leur plus grande qualité (178).

Qu'il s'agisse de « l'authentique » et du régional se référant au grand courant des échanges internationaux, de l'élévation des métiers d'artisanat aux métiers d'art (perçue par Hamel), c'est le même « rêve d'intégration » qui se laisse percevoir, autant à travers l'histoire et la malléabilité de ces textiles que dans les nouvelles orientations des ethnologues d'aujourd'hui.

D'autres articles encore mériteraient développement, notamment ceux de la quatrième partie, "Costume et mise en valeur ", ainsi que la cinquième partie qui devrait se révéler particulièrement utile aux muséologues, "Témoignages de collectionneurs ", transcription du débat sur les collections muséales orchestré lors du colloque sur les «Textiles d'Amérique et d'ailleurs ». Signalons seulement que l'ensemble de cet ouvrage est suffisamment dense pour que tout chercheur oeuvrant dans ce domaine y trouve une somme de références incontournables.

Et une remarque pour finir, particularité qui n'est pas la moindre : tous les auteurs de ce collectif sont des femmes. Le monde du textile resterait-il exclusivement féminin ? Quoiqu'il en soit, l'exercice interdisciplinaire est particulièrement réussi et devrait en encourager d'autres ; ici, histoire de l'art, muséologie, ethnologie, anthropologie, ethnohistoire se mêlent avec bonheur pour démontrer, si besoin était, que seule une approche globale et fluide, à la manière de celle-ci, peut permettre à l'objet (en l'occurrence ici les «arts textiles ») de livrer, sinon tout son sens, du moins le plus dense de ses significations.

L'ouvrage suivant réalise un gros-plan sur l'un des thèmes abordés par l'ouvrage précédent. Les broderies des Ursulines de Québec font partie de ces «textiles d'Amérique » que Le fil de l'art, catalogue raisonné réalisé par Christine Turgeon de l'exposition éponyme (Musée du Québec, 6 juin-17 novembre 2002), explore en une monographie exhaustive et minutieuse. Il faut signaler en tout premier lieu la remarquable qualité des illustrations tout en couleur de cet ouvrage, qui rendent hommage à la beauté de nombre de ces broderies ordinairement tenues loin des yeux du public, «entre la vie et le demisommeil », dans la chapelle du monastère des Ursulines (22). Le lecteur ne peut qu'être impressionné par l'extraordinaire finesse de ces enroulements baroques et fleuris, exploits techniques et esthétiques réalisés au petit point par des femmes discrètes, rarement donnés à voir 
et dont le déroulement coloré sur papier glacé sensibilise le profane au caractère sacré qui en émane.

L'auteure souligne l'intérêt de mener cette étude sur un ensemble de paramentique ${ }^{1}$ dont l'élaboration s'est déroulée en un lieu clos, sans bouleversement majeur, sur une période de près de quatre siècles, « loin des lieux de production traditionnels » (23), ce qui est une intention parfaitement classique, tout comme l'époque que l'auteure a choisi de détailler, la fin du XVII et le début du XVIII e siècle. Mais le souci des détails sensibles et l'exhaustivité permettent à l'auteure de découvrir la genèse, les choix délibérés et les influences qui vibrent et filtrent jusque dans l'enfermement du monastère, tout comme la participation réelle et consciente de ces femmes cloîtrées à la vie sociale et cérémonielle de leur temps.

Christine Turgeon a pris le parti d'analyser cette collection pour elle-même, dans ses lieux de production et ses lieux d'usage, à partir des broderies, des archives des Ursulines et des traditions orales des religieuses recueillies par Marius Barbeau dans les années 1940 (26). Il s'agit donc d'une vision de l'intérieur, qui permet à l'auteure de s'approcher au plus près, sans interférences, de la pensée, des personnalités et du regard sur leur temps de ces femmes qu'elle nous montre bien plus pragmatiques et ancrées dans leur société que les mystiques éthérées de l'imagerie qui s'attache aux religieuses de l'époque classique. Les religieuses évoquées par l'auteure dans son premier chapitre sont, comme la fondatrice du monastère, Marie de l'Incarnation, des femmes volontaires, ayant une expérience de vie et un remarquable sens pratique qu'elles appliquent avec détermination : leurs broderies délicates sont clairement appliquées à la bannière d'une «oeuvre de civilisation ». Dès le début, leurs ouvrages ont un rôle central dans les cérémonies et les processions qui appuient l'évangélisation. L'auteure remarque qu'elles ne s'inspirent jamais de «l'exotisme » du Nouveau Monde (qui est pourtant en Europe au même moment l'un des motifs décoratifs les plus en vogue) ; cette « oeuvre de civilisation» exige le rejet de l'imaginaire et de l'art autochtones. Lorsque, plus tardivement, elles intègrent à leur savoir-faire des techniques artistiques amérindiennes, c'est à titre anecdotique uniquement, pour fournir des «curiosités» en échange de quelques revenus (57). Elles établissent une démarcation nette entre ces arts profanes et la broderie " classique ",

1. Ornements d'autels, vêtement sacerdotaux. 
qui sacralise ce qu'elle orne. Les religieuses travaillent à la « vitrine de l'esthétique occidentale» (70) : elles fournissent les ornements pour les chapelles de mission et les parements cérémoniels. Leurs productions ont une fonction sociale de faste et de prestige et, bien qu'en retrait, elles accompagnent toute la vie publique de leur temps. La broderie fait également partie intégrante de l'oeuvre d'éducation des religieuses; c'est une discipline, une preuve de bonne éducation et un gagne pain, à des titres divers, pour les fillettes à qui elles transmettent leur savoirfaire.

Il y aurait beaucoup de détails très vivants, tout près des personnalités de ces femmes hors du monde et si présentes, à mentionner dans cet ouvrage. Retenons que l'auteur a démontré finement que cet ensemble de peintures à l'aiguille a été créé, élaboré, accumulé au fil du temps, dans l'enfermement du cloître, « en symbiose » avec l'histoire de la Nouvelle-France. Tout au long de l'étude, de magnifiques pièces anciennes éclairent le propos, gardant toujours en filigrane cette dimension «édifiante » de l'ouvrage des mains, ces mains qui élaborent patiemment, à force de finesse et de patience, l'entrée dans la dimension sacrée des choses qui doivent l'être. Les broderies des Ursulines sont une « oeuvre de civilisation» qui est aussi une oraison.

En résumé, ces deux livres sont, tout comme les splendides pièces d'étoffe dont ils traitent, tout comme ces prouesses de technique et d'esthétique que voudrait voir, plus souvent qu'on ne l'imagine, le public des musées... de la belle ouvrage. 